PROCEEDINGS OF THE

AMERICAN MATHEMATICAL SOCIETY

Volume 130, Number 2, Pages 599-607

S 0002-9939(01)06119-6

Article electronically published on July 25, 2001

\title{
FILLABLE CONTACT STRUCTURES ON TORUS BUNDLES OVER CIRCLES
}

\author{
PIOTR MIKRUT
}

(Communicated by Wolfgang Ziller)

\begin{abstract}
We will construct an example of a strongly symplectically fillable contact structure on a torus bundle over the circle with parabolic monodromy.
\end{abstract}

\section{INTRODUCTION}

In 1971 Martinet showed that each compact oriented 3-manifold admits a contact structure. The world of 3-dimensional contact manifolds is known to consist of two classes of manifolds, those with over twisted contact structures and those with tight contact structures. It is easy to construct an over twisted contact structure on a given contact manifold by applying the so-called Lutz twist. The class of over twisted contact 3-manifolds is classified by Eliashberg and the classification is equivalent to the classification of homotopy types of tangent plane fields of the manifold. Thus the only interesting contact manifolds as far as classification and the existence problem are concerned are the tight ones. Symplectic semifillability, fillability and strong fillability are notions related to contact manifolds and in dimension 3 all imply tightness. Here and in the next paper we give examples of manifolds which satisfy strong fillability and are torus bundles over a circle.

Definition 0.1. A 1 -form $\omega$ on a 3-manifold $M$ is contact if $\omega \wedge d \omega$ is nowhere equal to zero. The kernel of a contact manifold is a nowhere integrable tangent plane field on $M$. We say that a 3-manifold is a contact manifold if it has a globally defined nowhere integrable tangent plane field.

Definition 0.2. If $N$ is an embedded surface in $M$, then $\left.\omega\right|_{N}$ defines a foliation with singularities on $N$ called characteristic foliation. By an over twisted disk $D$ in a contact 3-manifold $M$ we mean an imbedded disk such that characteristic foliation on $D$ has a limit cycle on $\partial(D)$.

Definition 0.3. A contact structure on a 3-manifold $M$ represented by a contact form $\omega$ is called over twisted if there is an imbedded over twisted disk $D$ in $M$. Otherwise, the contact structure is called tight.

Received by the editors April 21, 2000 and, in revised form, July 31, 2000.

1991 Mathematics Subject Classification. Primary 57M60, 53C15.

Key words and phrases. 3-manifold, torus bundle, contact structure, tight contact structure, fillable contact structure, integrable systems.

The author was partially supported by the Polish Committee of Scientific Research grant 2 P03A 02314. 
Definition 0.4. A hypersurface $N$ in a symplectic 4-manifold $M$ is said to be of contact type if there is an expanding vector field $X$ near $N$, i.e. satisfying

$$
L_{X} \omega=\omega
$$

such that $i_{X} \omega$ is a contact form on $N$. If the hypersurface $N$ bounds a compact manifold $W$ in $M$ such that $X$ directs outside of $W$, then we say that the contact structure on $N$ is strongly symplectically fillable.

Let us note that each compact, contact 3-manifold is of contact type in its symplectization, i.e. in $N \times(-\infty, \infty)$ with symplectic form $d(t \omega)$. Here $t$ is a coordinate of $(-\infty, \infty)$. Thus $N$ bounds a symplectic manifold $N \times(-\infty, 0]$ which is noncompact. It is much more difficult for a contact 3-manifold to have strong symplectic filling.

For more basic information on contact manifolds see $\mathrm{ABKLR}, \overline{\mathrm{M}}$ and Et.

Theorem 0.5. $T^{2}$-bundle over a circle with parabolic monodromy

$$
\left(\begin{array}{ll}
1 & n \\
0 & 1
\end{array}\right)
$$

for natural number $n$ is strongly symplectically fillable.

Definition 0.6. Let a torus $T^{k_{\alpha}}$ act on a subset $U_{\alpha}$ of a contact 3-manifold $M$. Let $L$ denote the Lie derivative. Let $\theta_{\alpha}$ denote the action. Let $t_{\alpha}$ denote the space of vector fields on $U_{\alpha}$ generated by elements of the Lie algebra of $T^{k_{\alpha}}$. A contact form $\omega$ on $M$ is said to be invariant if

$$
L_{\xi}\left(\left.\omega\right|_{U_{\alpha}}\right)=0 .
$$

Here $M$ may be covered by open subsets like $U_{\alpha}$ with compatible actions. See the definition of an invariant contact structure on a manifold with $T$-structure in [Mi].

Filling of some $T^{2}$-bundles over $S^{1}$ with parabolic monodromies by the neighbourhood of singular fibres in elliptic fibrations. $T^{2}$-bundles over $S^{1}$ with $T^{2}$-invariant contact structures were studied by Giroux, Eliashberg and Kanda; see [Gi1], El1] and [Ka]. Here we give a symplectic filling of those with parabolic monodromies.

Let us first consider a $T^{2}$-bundle over $S^{1}$ with monodromy matrix

$$
\left(\begin{array}{ll}
1 & 1 \\
0 & 1
\end{array}\right)
$$

We will construct the symplectic filling of some $T^{2}$-invariant contact structure on the bundle. The filling is diffeomorphic to the neighbourhood of a singular fibre of type $I_{1}$ in an elliptic fibration; see [BPV] for suitable definitions. The notation $I_{1}$ is due to Kodaira. We will choose a symplectic structure on the filling so that the elliptic fibration becomes a singular Lagrangian fibration where the singular fibre corresponds to so-called focus-focus singularity studied by Nguyen-Tien-Zung ([NTZ]). In our construction we obtain a very explicit form of action-angle coordinates. The method of construction is related to toroidal embeddings; see section on Inoue surfaces in [0]. See also the closely related subject of constructing special Lagrangian fibrations on Elliptic K3 surfaces [BS]. 
Construction of the filling. Let $D=\left\{\left(z_{1}, z_{2}\right) \in C^{2}:\left|z_{1} z_{2}\right|<1\right\}$. Let $P$ be a transformation defined for $z_{2} \neq 0$ by the formula

$$
P\left(z_{1}, z_{2}\right)=\left(z_{2}^{-1}, z_{1}\left(z_{2}\right)^{2}\right) .
$$

Then $P\left(z_{1}, z_{2}\right)=\left(z_{1}, z_{2}\right)$ iff $z_{1} z_{2}=1$, i.e. $P$ has no fixed points on $D-\left\{z_{2}=0\right\}$. Let $D / P$ denote the quotient of $D$ by the equivalence relation

$$
\left(z_{1}, z_{2}\right) \sim\left(z_{1}^{\prime}, z_{2}^{\prime}\right) \text { iff }\left\{\left(z_{1}=z_{1}^{\prime}, z_{2}=z_{2}^{\prime}\right) \text { or }\left(z_{2} \neq 0 \text { and }\left(z_{1}^{\prime}, z_{2}^{\prime}\right)=P\left(z_{1}, z_{2}\right)\right)\right\} .
$$

Let $z_{1}=r_{1} e^{i \phi_{1}}, z_{2}=r_{2} e^{i \phi_{2}}$. Then, for $r_{2} \neq 0$,

$$
\begin{gathered}
P\left(r_{1} e^{i \phi_{1}}, r_{2} e^{i \phi_{2}}\right)=\left(\frac{1}{r_{2}} e^{-i \phi_{2}}, r_{1} r_{2}^{2} e^{i\left(\phi_{1}+2 \phi_{2}\right)}\right), \\
P\left(0, z_{2}\right)=\left(z_{2}^{-1}, 0\right), \\
P^{-1}\left(z_{1}, z_{2}\right)=\left(z_{1}^{2} z_{2}, z_{1}^{-1}\right) .
\end{gathered}
$$

It is now easy to see that $D / P$ is a complex manifold with the complex structure inherited from $D$. Moreover, $f\left(z_{1}, z_{2}\right):=z_{1} z_{2}$ is a holomorphic function on $D / P$ and $\Omega:=d z_{1} \wedge d z_{2}$ is a holomorphic 2-form. Let $\omega=\operatorname{Re}\left(d z_{1} \wedge d z_{2}\right)$. Then $\omega$ is a symplectic form on $D / P$ such that $\omega \wedge \omega$ defines the orientation the same as that given by the complex structure. Let

$$
z_{1}=x_{1}+i y_{1} \quad z_{2}=x_{2}+i y_{2} ;
$$

then

$$
\Omega=d z_{1} \wedge d z_{2}=d x_{1} \wedge d x_{2}-d y_{1} \wedge d y_{2}+i\left(d y_{1} \wedge d x_{2}+d x_{1} \wedge d y_{2}\right) .
$$

Thus

$$
\omega=d x_{1} \wedge d x_{2}-d y_{1} \wedge d y_{2}
$$

and

$$
\omega \wedge \omega=2\left(d x_{1} \wedge d y_{1} \wedge d x_{2} \wedge d y_{2}\right) .
$$

In real coordinates we have

$$
f\left(x_{1}, x_{2}, y_{1}, y_{2}\right)=z_{1} z_{2}=x_{1} x_{2}-y_{1} y_{2}+i\left(y_{1} x_{2}+x_{1} y_{2}\right) .
$$

The derivative of $f$ is given by the matrix

$$
\left(\begin{array}{cccc}
x_{2} & x_{1} & -y_{2} & -y_{1} \\
y_{2} & y_{1} & x_{2} & x_{1}
\end{array}\right) .
$$

In the complex variables $d f=z_{1} d z_{2}+z_{2} d z_{1}$. The rank of the matrix is equal to 2 for $\left(x_{1}, x_{2}, y_{1}, y_{2}\right) \neq(0,0,0,0)$. In particular, we obtain that $f^{-1}(c)$ for $c \neq 0$ is a complex submanifold of $D / P$. $f^{-1}(0)$ is an immersed 2-sphere, locally a complex submanifold of $D / P$.

Moreover, on $f^{-1}(c)$ we have $z_{1} z_{2} \equiv c$ and $d f=z_{1} d z_{2}+z_{2} d z_{1}=0$. We can calculate for $z_{2} \neq 0$,

$$
\left.\Omega\right|_{f^{-1}(c)}=d z_{1} \wedge\left(-\frac{z_{2}}{z_{1}} d z_{1}\right)=0 .
$$

In particular, from continuity of $\omega$,

$$
\left.\omega\right|_{f^{-1}(c)}=0 .
$$


Analogously,

$$
\left.\Omega\right|_{f^{-1}(0)}=0
$$

and

$$
\left.\omega\right|_{f^{-1}(0)}=0
$$

on a manifold part of $f^{-1}(0)$. Thus $f^{-1}(c)$ are Lagrangian submanifolds of the symplectic manifold $(D / P, \omega)$.

It can be easily checked that $\operatorname{Re}(f)$ and $\operatorname{Im}(f)$ are Poisson commuting functions. Let us fix notation

$$
f_{X_{1}}:=\operatorname{Re}(f), \quad f_{X_{2}}:=\operatorname{Im}(f)
$$

where $X_{1}, X_{2}$ are Hamiltonian vector fields defined by

$$
d\left(f_{X_{1}}\right)=i_{X_{1}} \omega, \quad d\left(f_{X_{2}}\right)=i_{X_{2}} \omega .
$$

Since

$$
x_{1} d x_{2}+x_{2} d x_{1}-y_{1} d y_{2}-y_{2} d y_{1}=i_{X_{1}} \omega
$$

and

$$
y_{2} d x_{1}+x_{1} d y_{2}+y_{1} d x_{2}+x_{2} d y_{1}=i_{X_{2}} \omega,
$$

we can compute for $r_{1} \neq 0, r_{2} \neq 0$ :

$$
\begin{gathered}
X_{1}=x_{1} \partial_{x_{1}}-x_{2} \partial_{x_{2}}+y_{1} \partial_{y_{1}}-y_{2} \partial_{y_{2}}=r_{1} \partial_{r_{1}}-r_{2} \partial_{r_{2}}, \\
X_{2}=y_{1} \partial_{x_{1}}-y_{2} \partial_{x_{2}}-x_{1} \partial_{y_{1}}+x_{2} \partial_{y_{2}}=\partial_{\phi_{1}}-\partial_{\phi_{2}} .
\end{gathered}
$$

In particular, $X_{2}$ generates the Hamiltonian action of $S^{1}$ with Hamiltonian function $-f_{X_{2}}$.

The set $f^{-1}(c)$ for $0<|c|<1$ is parametrized by $(r, \phi)$, where $\left(z_{1}, z_{2}\right)=$ $\left(c r e^{i \phi}, \frac{1}{r} e^{-i \phi}\right)$, i.e.

$$
r=\frac{1}{\left|z_{2}\right|}, \quad \phi=\operatorname{Arg} \frac{1}{z_{2}} .
$$

$P$ acts by

$$
(r, \phi) \longmapsto\left(\frac{r}{|c|}, \phi-\operatorname{Arg}(c)\right) .
$$

Thus $f^{-1}(c)$ for $0<|c|<1$ is diffeomorphic to $T^{2}$. From the Arnold-Liouville theorem we can expect existence of action-angle coordinates in the neighbourhood of $f^{-1}(c)$ in $D / P$.

The flow of $X_{1}$ is given by the formula

$$
\left(z_{1}, z_{2}\right) \longmapsto\left(e^{s} z_{1}, e^{-s} z_{2}\right) .
$$

In coordinates $r, \phi$ it is given by

$$
(r, \phi) \longmapsto\left(r e^{s}, \phi\right) .
$$

Let

$$
Y_{1}:=\left(\frac{1}{2 \pi} \log \frac{1}{|c|}\right) X_{1}=\left(\frac{1}{2 \pi} \log \frac{1}{\left|z_{1} z_{2}\right|}\right) X_{1} .
$$

The flow of $Y_{1}$ after a time $2 \pi$ acts by

$$
(r, \phi) \longmapsto\left(\frac{r}{\left|z_{1} z_{2}\right|}, \phi\right) .
$$


Let us compare it to the action of $P$ :

$$
(r, \phi) \longmapsto\left(\frac{r}{\left|z_{1} z_{2}\right|}, \phi-\operatorname{Arg}\left(z_{1} z_{2}\right)\right) .
$$

Let

$$
Y_{2}=\frac{\operatorname{Arg}\left(z_{1} z_{2}\right)}{2 \pi} X_{2}
$$

then the flow of $Y_{2}$ after a time $2 \pi$ acts by

$$
(r, \phi) \longmapsto\left(r, \phi-\operatorname{Arg}\left(z_{1} z_{2}\right)\right) .
$$

Let

$$
Y_{3}:=Y_{1}+Y_{2}=\frac{1}{2 \pi}\left(\left(\log \frac{1}{\left|z_{1} z_{2}\right|}\right) X_{1}+\operatorname{Arg}\left(z_{1} z_{2}\right) X_{2}\right) .
$$

Thus the flow of $Y_{3}:=Y_{1}+Y_{2}$ after a time $2 \pi$ acts by

$$
(r, \phi) \longmapsto\left(\frac{r}{\left|z_{1} z_{2}\right|}, \phi-\operatorname{Arg}\left(z_{1} z_{2}\right)\right) .
$$

The formula is the same as for $P$.

Let us note that $X_{1}, X_{2}$ commute in the sense of the Poisson bracket

$$
\left\{X_{1}, X_{2}\right\}=0
$$

and in the sense of the Lie bracket

$$
\left[X_{1}, X_{2}\right]=0 .
$$

Similarly, we obtain

$$
\left\{Y_{3}, X_{2}\right\}=0, \quad\left[Y_{3}, X_{2}\right]=0 .
$$

The flow of $Y_{3}$ after a time $s$ acts by

$$
(r, \phi) \longmapsto\left(r e^{\left(\frac{1}{2 \pi} \log \frac{1}{z_{1} z_{2}}\right) s}, \phi-\frac{\operatorname{Arg}\left(z_{1} z_{2}\right)}{2 \pi} s\right)
$$

or in coordinates $z_{1}, z_{2}$

$$
\begin{gathered}
z_{1} \longmapsto\left(z_{1} z_{2}\right)^{1-\frac{s}{2 \pi}} z_{2}^{-1}, \\
z_{1} \longmapsto\left(z_{1} z_{2}\right)^{\frac{s}{2 \pi}} z_{2} .
\end{gathered}
$$

In particular, it preserves $\Omega$.

If $z_{1} z_{2}=R e^{i \psi}$ and we change the argument $\psi$ by $2 \pi$ in a positive direction, then we can observe the action of the monodromy transformation on $Y_{3}$

$$
Y_{3} \longmapsto Y_{3}+X_{2} \text {. }
$$

Let us define $f_{Y_{3}}$ by the formula

$$
d\left(f_{Y_{3}}\right)=i_{Y_{3}} \omega
$$

then we can choose

$$
f_{Y_{3}}:=\frac{1}{2 \pi} \operatorname{Re}\left(z_{1} z_{2}\left(1-\log \left(z_{1} z_{2}\right)\right)\right) .
$$

In coordinates $R, \psi(0<R<1,0 \leq \psi<2 \pi)$

$$
\begin{gathered}
f_{X_{2}}=R \sin (\psi) \\
f_{Y_{3}}=\frac{1}{2 \pi}(R \cos (\psi)(1-\log R)+R \psi \sin (\psi)) .
\end{gathered}
$$


We can check that

$$
\frac{\partial}{\partial \psi}\left(\frac{f_{Y_{3}}}{f_{X_{2}}}\right)<0
$$

The function $\frac{f_{Y_{3}}}{f_{X_{2}}}$ has value 0 for two values of $\psi \in[0,2 \pi]$. For $\psi=0, \pi$ we obtain vertical asymptote of the function. The image of the singular Lagrangian fibration defined by $f$ represents an affine disk with one singular point for $R=0$. The affine structure is defined by locally defined coordinates $Y_{3}, X_{2}$. From the behaviour of these coordinates it follows that we can construct such a disk from a standard unit disk on $R^{2}$

$$
B=\left\{(x, y): x^{2}+y^{2}<1\right\}
$$

by removing the sector

$$
\left\{(x, y): x^{2}+y^{2}<1, x>0, y>0, x>y\right\}
$$

and gluing the intervals

$$
\begin{aligned}
& \left\{(x, y): x^{2}+y^{2}<1, x \geq 0, y=0\right\}, \\
& \left\{(x, y): x^{2}+y^{2}<1, x \geq 0, y=x\right\}
\end{aligned}
$$

using the affine map

$$
(x, y) \longmapsto(x, x+y) .
$$

We can also check that

$$
\omega=d H_{1} \wedge d t+d H_{2} \wedge d s
$$

where

$$
H_{1}=-f_{X_{2}}, \quad H_{2}=-f_{Y_{3}}
$$

are action coordinates and

$$
\begin{gathered}
s:=\pi \frac{\left.\log \left(\left|z_{2}\right|\right)-\log \left(\left|z_{1}\right|\right)\right)}{\log \left(\left|z_{1}\right|\right)+\log \left(\left|z_{2}\right|\right)}=\pi \frac{\log \left(\frac{r_{2}}{r_{1}}\right)}{\log \left(r_{1} r_{2}\right)}, \\
t:=\frac{\operatorname{Arg}\left(z_{2}\right)-\operatorname{Arg}\left(z_{1}\right)}{2}-\frac{s}{2 \pi}\left(\operatorname{Arg}\left(z_{1}\right)+\operatorname{Arg}\left(z_{2}\right)\right) \\
=\frac{\phi_{2}-\phi_{1}}{2}-\frac{s}{2 \pi}\left(\phi_{1}+\phi_{2}\right)
\end{gathered}
$$

are angle coordinates. The monodromy is given by the formula

$$
\left(H_{1}, H_{2}, t, s\right) \longmapsto\left(H_{1}, H_{1}+H_{2}, t-s, s\right) .
$$


The submanifold defined by $R=$ const is of contact-type. Let

$$
X:=H_{1} \partial_{H_{1}}+H_{2} \partial_{H_{2}} .
$$

Then $X$ is globally defined on $D / P$ since the formula for $X$ is preserved by monodromy. $X$ satisfies

$$
L_{X} \omega=\omega
$$

The 1-form

$$
\alpha=i_{X} \omega=H_{1} \wedge d t+H_{2} \wedge d s
$$

restricted to the submanifold $N$ defined by $0<R=R_{0}<1$ is a $T^{2}$-invariant contact form and the symplectic manifold defined by $R \leq R_{0}$ is a strong symplectic filling of $(N, \alpha)$. The filling is compact since topologically it is a closed neighbourhood of a singular fibre of type $I_{1}$ in elliptic fibration or a singular fibre of focus-focus type in singular Lagrangian torus fibration with generic fibre $T^{2}$. The singular locus is the so-called fishtail singularity.

The fundamental group of the filling just constructed is isomorphic to $Z$. Let us take a connected $n$-fold covering space. Its boundary is the $T^{2}$-bundle over $S^{1}$ with monodromy matrix

$$
\left(\begin{array}{ll}
1 & n \\
0 & 1
\end{array}\right)
$$

We obtained a symplectic filling of the $T^{2}$-invariant contact structure on the bundle which is induced from $\alpha$. The filling is diffeomorphic to the neighbourhood of a singular fibre of type $I_{n}$ in the terminology of Kodaira. The filling is a singular Lagrangian fibration with $n$ singular point with focus-focus singularity. The actionangle coordinates are represented by the locally defined functions $\left(H_{1}, H_{2}, t, s\right)$ on the covering. $X_{2}$ generates $S^{1}$ action on $D / P$ with one fixed point in $\left(z_{1}, z_{2}\right)=$ $(0,0)$. Thus the generic orbit of $X_{2}$ is contractible in $D / P$. The local Hamiltonian $H_{2}$ generates locally an $S^{1}$ action. A generic orbit of this action represents a generator of $\pi_{1}(D / P)$. Since the filling of $N$ is diffeomorphic to $D / P$, the orbit also represents a generator of the fundamental group of the filling. Thus on the $n$-fold covering, the coordinate $s$ is periodic with period $2 \pi n$. Let us put

$$
s^{\prime}=\frac{s}{n}, \quad H_{2}^{\prime}=n H_{2},
$$

then $t$ and $s^{\prime}$ have period $2 \pi$ on the covering. The induced symplectic form is defined by the same formula

$$
\omega=d H_{1} \wedge d t+d H_{2}^{\prime} \wedge d s^{\prime}
$$

and the monodromy is given by

$$
\left(H_{1}, H_{2}^{\prime}, t, s^{\prime}\right) \longmapsto\left(H_{1}, n H_{1}+H_{2}^{\prime}, t-n s^{\prime}, s^{\prime}\right) .
$$

We can construct the covering by means of plumbing $n$ disk bundles over $S^{2}$ with self-intersection numbers -2 according to orientation given by the complex structure. 
Construction of the filling in another way. Let us consider a holomorphic $D^{2}$ bundle over $C P^{1}=S^{2}$ with Euler number -2 . Let $z_{1}$ be a complex coordinate on a neighbourhood of southern hemisphere and $z_{1}^{\prime}$ a complex coordinate on a neighbourhood of nothern hemisphere. Then near the equator,

$$
z_{1}=\frac{1}{z_{1}^{\prime}}
$$

Let $z_{2}$ be a coordinate of a fibre $D^{2}$ over a neighbourhood of the southern hemisphere and $z_{2}^{\prime}$ a coordinate of a fibre $D^{2}$ over a neighbourhood of the northern hemisphere. Then over the neighbourhood of the equator,

$$
z_{2}^{\prime}=-z_{1}^{2} z_{2} \text {. }
$$

We can check easily that the holomorphic 2 -form written in local coordinates by

$$
\Omega=d z_{1} \wedge d z_{2} \quad \text { or } \quad \Omega=d z_{1}^{\prime} \wedge d z_{2}^{\prime}
$$

is globally defined on the total space of the fibration. In particular, the real 2-form

$$
\omega:=\operatorname{Re}(\Omega)
$$

is a symplectic 2 -form. The zero section of the fibration is a Lagrangian sphere and the total space of the bundle is symplectomorphic to a subset of a cotangent bundle of $S^{2}$ with a canonical 2-form. The function

$$
G\left(z_{1}, z_{2}\right)=z_{1} z_{2} \quad \text { or } \quad G\left(z_{1}^{\prime}, z_{2}^{\prime}\right)=-z_{1}^{\prime} z_{2}^{\prime}
$$

is a holomorphic function on the fibration. Preimages of points are Lagrangian submanifolds.

Let us take $n$ copies of such fibrations and let us denote local coordinates $\left(z_{1, i}, z_{2, i}, z_{1, i}^{\prime}, z_{2, i}^{\prime}\right)$ for $i=1, \ldots, n$ correspondingly. Let us plumb them using coordinate transformations

$$
z_{1, i}=-z_{2, i+1}^{\prime}, \quad z_{2, i}=z_{1, i+1}^{\prime}
$$

where $n+1$ means 1 . Then the holomorphic 2 -form $\Omega$ and the function $G$ are well defined on the plumbing. The cyclic group $Z_{n}$ acts on the obtained manifold by identifying a point with coordinates $z_{1, i}, z_{2, i}$ or $z_{1, i}^{\prime}, z_{2, i}^{\prime}$ with the point having coordinates $z_{1, i+1}, z_{2, i+1}$ or $z_{1, i+1}^{\prime}, z_{2, i+1}^{\prime}$ correspondingly where $z_{1, i}=z_{1, i+1}, z_{2, i}=$ $z_{2, i+1}$ or $z_{1, i}^{\prime}=z_{1, i+1}^{\prime}, z_{2, i}^{\prime}=z_{2, i+1}^{\prime}$. The quotient of the submanifold $|G|<1$ by the action of $Z_{n}$ can be identified with $D / P$ constructed earlier.

Using known constructions of singular fibres in elliptic fibrations we extend in the next paper our argument to other $T^{2}$-bundles over $S^{1}$.

\section{ACKNOWLEDGEMENTS}

The author was inspired by reading the papers or books of Margaret Symington, John Etnyre, Bob Gompf, András Stipsicz, Emmanuel Giroux, Nguyen-Tien-Zung. I would like to thank the authors.

\section{REFERENCES}

[ABKLR] B.Aebischer, M.Borer, M.Kälin, Ch.Leuenberger, H.M.Reimann, Symplectic Geometry, Birkhäuser Basel, Boston, Berlin, (1994). MR 96a:58082

[BPV] W.Barth, C.Peters, A.Van der Ven, Compact Complex Surfaces, Springer-Verlag (1984). MR 86c:32026

[BS] Uga Bruzzo, Guido Sanguinetti, Mirror symmetry on K3 surfaces as a hyper-Käeler rotation, Lett.Math.Phys. 45 (1998), 295-301. MR 99k:14064 
[Et] John B.Etnyre, Symplectic convexity in low-dimensional topology, Proceedings of the Georgia Conference Topology Conference (1996), Topology and its Applications 88 no. 1-2 (1998), 3-25. MR 99j:57014

[El1] Y.Eliashberg,, Unique holomorphically fillable contact structures on the 3-torus, Int. Math. Res. Notices 2 (1996), 77-82. MR 97b:53034

[Gi1] E.Giroux, Une structure de contact, meme tendue est plus ou moins tordue, Ann. Scient. Ec. Norm. Sup. 27 (1994), 697-705. MR 96b:57034

[Ka] Y.Kanda, The classification of tight contact structures on the 3-torus, Comm. Anal. Geom. 5 (1997), 413-438. MR 99c:57054

[M] J.Martinet, Formes de contact sur les variétés de dimension 3, Springer Lecture Notes in Math. 209, (1971), 142-163. MR 50:3263

[Mi] P.Mikrut, Invariant contact structure on 3-manifolds with local actions of tori, preprint.

[NTZ] Nguyen-Tien-Zung, Symplectic topology of integrable Hamiltonian systems, I: ArnoldLiouville with singularities, Compositio Mathematica, 101 (1996), 179-215. MR 97c:58052

[O] T.Oda, Lectures on torus embeddings and its applications, Springer-Verlag, Tata Institute of Fundamental Research, Bombay (1978). MR 81e:14001

Mathematical Institute, University of WrocŁaW, Pl.Grunwaldzki 2/4, 50-384 WroCEAW, Poland

E-mail address: mikrut@math.uni.wroc.pl 